2009

\title{
Magnetic Reconnection In Two-Dimensional Magnetohydrodynamic Turbulence
}

S. Servidio

W. H. Matthaeus

M. A. Shay

P. A. Cassak

P. Dmitruk

Follow this and additional works at: https://researchrepository.wvu.edu/faculty_publications

\section{Digital Commons Citation}

Servidio, S.; Matthaeus, W. H.; Shay, M. A.; Cassak, P. A.; and Dmitruk, P., "Magnetic Reconnection In Two-Dimensional Magnetohydrodynamic Turbulence" (2009). Faculty Scholarship. 589.

https://researchrepository.wvu.edu/faculty_publications/589 


\title{
Magnetic Reconnection in Two-Dimensional Magnetohydrodynamic Turbulence
}

\author{
S. Servidio, ${ }^{1}$ W. H. Matthaeus, ${ }^{1}$ M. A. Shay, ${ }^{1}$ P. A. Cassak, ${ }^{2}$ and P. Dmitruk ${ }^{1,3}$ \\ ${ }^{1}$ Bartol Research Institute and Department of Physics and Astronomy, University of Delaware, Newark, Delaware 19716, USA \\ ${ }^{2}$ Department of Physics, West Virginia University, Morgantown, West Virginia 26506, USA \\ ${ }^{3}$ Departmento de Física, Facultad de Ciencias Exactas y Naturales, Universidad de Buenos Aires, \\ Ciudad Universitaria, 1428, Buenos Aires, Argentina \\ (Received 10 December 2008; published 18 March 2009)
}

\begin{abstract}
Systematic analysis of numerical simulations of two-dimensional magnetohydrodynamic turbulence reveals the presence of a large number of $X$-type neutral points where magnetic reconnection occurs. We examine the statistical properties of this ensemble of reconnection events that are spontaneously generated by turbulence. The associated reconnection rates are distributed over a wide range of values and scales with the geometry of the diffusion region. Locally, these events can be described through a variant of the Sweet-Parker model, in which the parameters are externally controlled by turbulence. This new perspective on reconnection is relevant in space and astrophysical contexts, where plasma is generally in a fully turbulent regime.
\end{abstract}

DOI: 10.1103/PhysRevLett.102.115003

The plasma dynamical processes leading to changes of magnetic topology and release of magnetic energy are collectively called magnetic reconnection [1-3]. These processes are of fundamental importance in space [4], astrophysical [5], and laboratory settings [6]. Magnetic reconnection is often studied in simplified geometries and boundary conditions [7,8], but since it might occur in any region separating topologically distinct magnetic flux structures, it might be expected to be of importance in more general circumstances, including magnetohydrodynamic (MHD) turbulence [9]. It is, in fact, difficult to envision a turbulent cascade without change of magnetic topology. Furthermore, turbulence may provide a kind of unbiased or natural local boundary condition that provides a view of reconnection relatively unaffected by arbitrary (imposed) conditions. Although some suggestions have been made concerning both the general role of reconnection in MHD turbulence [10-12] and the impact of turbulence on reconnection of large structures $[9,10,13]$, no quantitative description of these effects has been documented, especially at high Reynolds numbers.

Here we examine the statistical properties of thousands of incompressible MHD reconnection events occurring at $X$-type neutral points at large magnetic and fluid Reynolds numbers, using direct numerical simulations with resolutions up to $8192^{2}$ grid points. We find a broad range of reconnection rates, reaching as high as $\simeq 0.3$ normalized to the root-mean-square magnetic field. A characteristic power-law distribution of rates is found relative to the geometrical aspect ratio of the reconnecting sites. We will examine how these surprising features can be brought into accord with a generalized form of classical SweetParker scenario.

The premise of the present study is that reconnection in the midst of turbulence responds to the complex conditions established by the nonlinear cascade, a scenario that differs
PACS numbers: 52.35.Vd, 96.50.Bh, 96.50.Tf, 96.60.Iv

from the familiar symmetric and isolated models in which reconnection is often studied [7-10,13,14]. A threedimensional treatment of the type we undertake is unfeasible at present, so we proceed using two-dimensional 2D MHD models, which can attain higher spatial resolution.

The 2D incompressible MHD equations can be written in terms of the magnetic potential $a(x, y)$ and the stream function $\phi(x, y)$ (uniform mass density $\rho=1$ ) as follows [15]:

$$
\begin{gathered}
\frac{\partial \omega}{\partial t}=-(\boldsymbol{v} \cdot \boldsymbol{\nabla}) \omega+(\boldsymbol{b} \cdot \boldsymbol{\nabla}) j+R_{\nu}^{-1} \nabla^{2} \omega, \\
\frac{\partial a}{\partial t}=-(\boldsymbol{v} \cdot \boldsymbol{\nabla}) a+R_{\mu}^{-1} \nabla^{2} a,
\end{gathered}
$$

where the magnetic field is $\boldsymbol{b}=\boldsymbol{\nabla} \boldsymbol{a} \times \hat{\boldsymbol{z}}$, the velocity $\boldsymbol{v}=$ $\boldsymbol{\nabla} \phi \times \hat{z}$, the current density $j=-\nabla^{2} a$, and the vorticity $\omega=-\nabla^{2} \phi$. Equations (1) and (2) are written in familiar Alfvén units [9] with lengths scaled to $L_{0} . R_{\mu}$ and $R_{\nu}$ are magnetic and kinetic Reynolds numbers at scale $L_{0}$, respectively.

Equations (1) and (2) are solved in doubly periodic $(x, y)$ Cartesian geometry (side $\left.2 \pi L_{0}\right)$, with a dealiased $(2 / 3$ rule) pseudospectral code [16]. We report results from runs with up to $8192^{2}$ grid points and $R_{\nu}=R_{\mu}=5000$. Time integration is second order Runge-Kutta. The initial state consists of uncorrelated, equipartitioned velocity, and magnetic field fluctuations, with random phases. For the main $8192^{2}$ run, energy is initially in the shell $5 \leq k \leq 30$ ( $k$ in units of $1 / L_{0}$ ). Total energy is $E=\left\langle|\boldsymbol{v}|^{2}+|\boldsymbol{b}|^{2}\right\rangle \simeq 1$ $(\langle\ldots\rangle$ denotes a spatial average). The time of our analysis is at the peak of the mean-square current density $\left\langle j^{2}\right\rangle$, when nonlinear activity is strong $(t \sim 0.3)$. Figure 1 (a) shows that coherent structures appear in the current, $j$, which is intermittently intense as expected in MHD turbulence $[10,12]$. The numerical results shown were verified in a number of ways: We compared runs with different time 


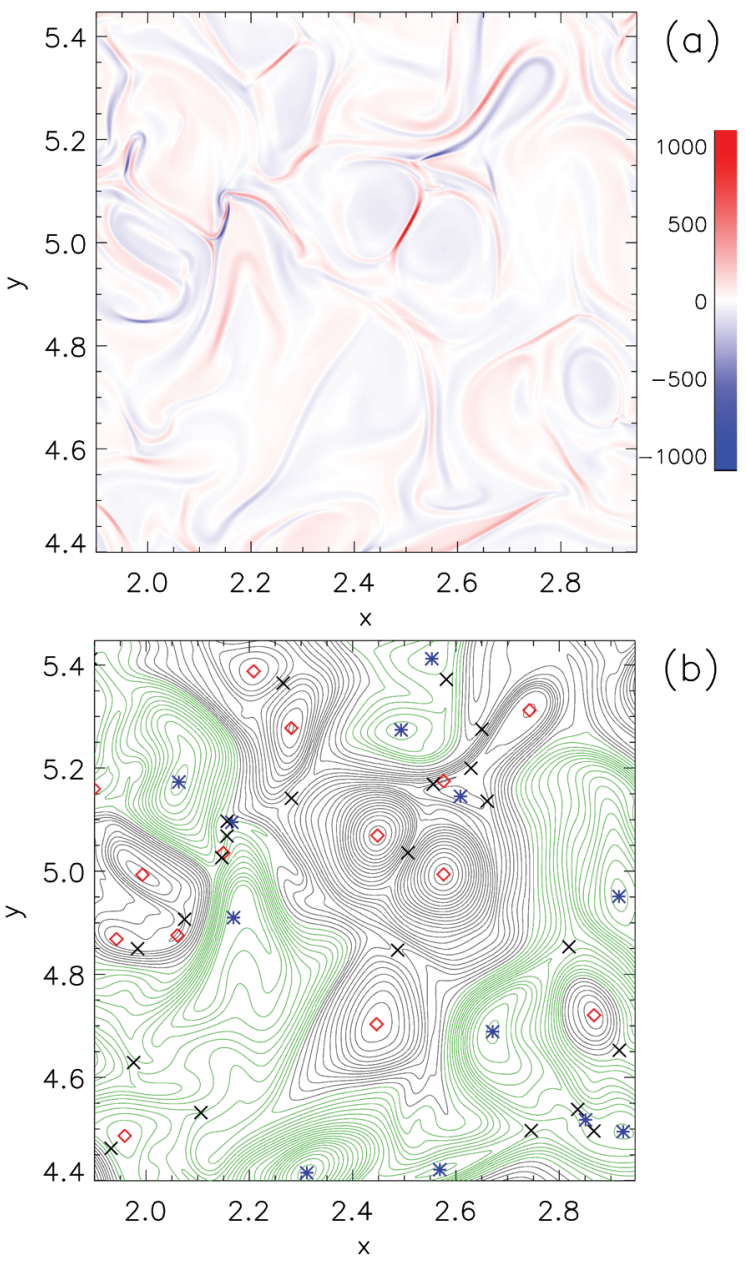

FIG. 1 (color). (a) Color map of $j$. (b) Contours of magnetic potential $a$ ( $a>0$ green, $a<0$ black), with the positions of maximum (blue stars), minimum (red open squares) and $X$ points (black $\times$ ). Only one-sixth of the box is shown.

steps and spatial resolutions, checked that the dissipation scale is resolved, and examined field lines at the grid scale for adequate smoothness.

To understand the magnetic field topology we inspect $a(x, y)$ [15]. The square Hessian matrix of $a$ is $H_{i, j}^{a}(\boldsymbol{x})=$ $\frac{\partial^{2} a}{\partial x_{i} \partial x_{j}}$ [17]. At each neutral point, $\nabla a=0$, we compute the eigenvalues of $H_{i, j}^{a}$. If both eigenvalues are positive (negative), the point is a local minimum (maximum) of $a$ (an $O$ point). If the eigenvalues are of mixed sign, it is a saddle point (an $X$ point) [17]. Figure 1(b) shows an example of a magnetic potential landscape together with its critical points. The number of $X$ points is $\simeq 1300$. A sea of magnetic islands, different in size and energy, is present. These coherent structures interact nonlinearly, merge, stretch, attract, and repulse each other. At the boundaries of these magnetic islands, the burstiness of $j$ reflects the intermittent nature of the magnetic field [10]. In these (diffusion) regions reconnection is expected.

The local geometry of the diffusion region is related to the Hessian eigenvalues

$$
\lambda_{\max }=\frac{\partial^{2} a}{\partial \eta^{2}}, \quad \lambda_{\min }=\frac{\partial^{2} a}{\partial l^{2}},
$$

the larger and smaller (in magnitude), respectively, and the associated unit eigenvectors $\hat{s}_{\eta}$ and $\hat{s}_{l}$. The coordinate $\eta$ is associated with the minimum thickness $\delta$ of the current sheet, while $l$ with the elongation $\ell$. From a scaling analysis of Eq. (3), the aspect ratio of the diffusion region is well approximated by

$$
\frac{\ell}{\delta} \simeq \sqrt{\lambda_{R}}, \quad \text { where } \lambda_{R}=\left|\frac{\lambda_{\max }}{\lambda_{\min }}\right| .
$$

After finding all potential reconnection zones, we evaluate the associated time rate of change of the magnetic flux $\frac{\partial a}{\partial t} \equiv \dot{a}$. From Eq. (2), at each $X$ point, the rate is

$$
\dot{a}=\left.R_{\mu}^{-1} \nabla^{2} a\right|_{X \text {-point }}=-E_{\times} .
$$

The reconnection rate $E_{\times}$is normalized to the root-meansquare magnetic fluctuation $\delta b_{\mathrm{rms}}^{2}$, as is appropriate to dimensionless Alfvén units. Figure 2 shows that the reconnection rates are broadly distributed with a range $\left|E_{\times}\right| \in$ $\left[10^{-6}-0.3\right]$ with $\left\langle\left|E_{\times}\right|\right\rangle \simeq 0.05$.

If reconnection is in a steady state, the rate depends on the aspect ratio defined by Eq. (4). In Fig. 2, the extreme (strongest) reconnection rates scale as

$$
E_{\times} \sim \frac{\ell}{\delta} .
$$

This behavior is counterintuitive in the context of standard Sweet-Parker theory, where $E_{\times} \sim \delta / \ell$ [1]. We will return to this issue later.

The power law in Fig. 2 supports Eq. (6) only for the fastest reconnection events. For more slowly reconnecting regions there is no clear scaling. The distinguishing characteristic of the strong reconnection sites appears to be a near-steady interaction between larger, more energetic magnetic islands. To select these we set a threshold for $E_{\times}$and $\lambda_{R}$. We can further understand the strong events by

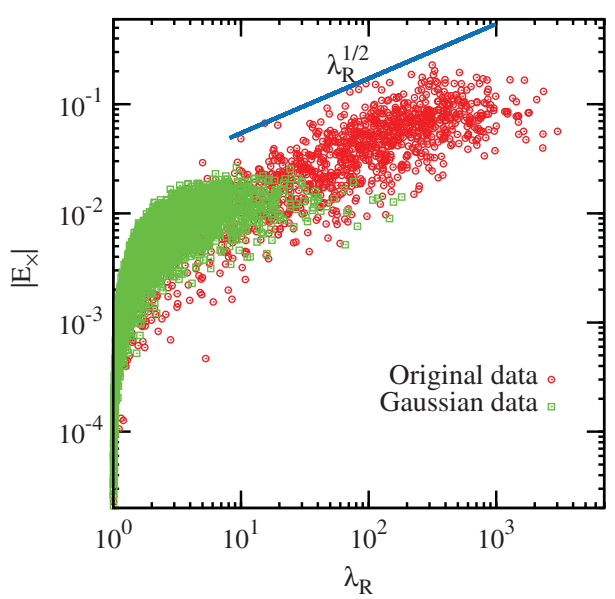

FIG. 2 (color). The reconnection rates $E_{\times}$vs the aspect ratio of the diffusion region $\lambda_{R}$ (red dots). The power-law fit (blue line, shifted in the $y$ axis) suggests $E_{\times} \sim(\ell / \delta)$. The Gaussianized field (green squares) is shown for comparison. 
comparison with a hybrid sample in which the coherency is destroyed. The nonlinearity of MHD gives rise to coherency, which is hidden in the phases of the Fourier coefficients. To destroy it, we Fourier transform $a(\boldsymbol{x})$, obtaining $\tilde{a}(\boldsymbol{k})$, and we randomize the phases retaining the same spectrum $|\tilde{a}(\boldsymbol{k})|^{2}$ [18]. An inverse Fourier transform provides the incoherent Gaussianized function $a^{\text {Gauss }}(\boldsymbol{x})$. Figure 2 shows that the reconnection rates for $a^{\text {Gauss }}$ are much lower than for the original field: this is a strong indication that turbulence enhances reconnection [10]. This can be very important in the context of particle acceleration problems, where a random-phase approximation may fail as a realistic description of a turbulent plasma [19]. The threshold for $E_{\times}$and $\lambda_{R}$ is given by the limits suggested by the Gaussianized field. From analysis of the data in Fig. 2, we use the thresholds $E_{\times}>0.013$ and $\lambda_{R}>100$. In the following, we will use only data exceeding these thresholds, and show these are quasistationary events.

At this point we characterize every diffusion region including determination of $\delta$ and $\ell$. We know the eigenvalue ratios, so the problem reduces to finding $\delta[\ell$ can be obtained by Eq. (4)]. At each $X$ point, using a system of reference given by the unit vectors $\left\{\hat{s}_{\eta}, \hat{s}_{l}\right\}$, we compute normal and tangential components of the magnetic field as $b_{n}=\boldsymbol{b} \cdot \hat{s}_{\eta}$ and $b_{t}=\boldsymbol{b} \cdot \hat{s}_{l}$, respectively. The magnetic field profile across the current sheet in the strongest reconnection events reaches a maximum and then decreases far from the saddle point $[20,21]$. In slowly reconnecting cases the magnetic field increase is monotonic. This differs from standard models where often the upstream magnetic field is uniform.

The simulation data demonstrate that reconnection in turbulence takes place in typically asymmetric regions with unequal field strengths in the two upstream regions. This implies that the peak of the current density is not centered at the $X$ point $[22,23]$. The diffusion region can be divided in adjacent sides of width $\delta_{1}$ and $\delta_{2}\left(\delta=\delta_{1}+\right.$ $\delta_{2}$ ), found by fitting with hyperbolic functions. This determines the current sheet width, and at this position we evaluate the upstream magnetic field. A histogram of the distributions of $\delta$ and $\ell$ is reported in Fig. 3(a). For this run we found $\langle\delta\rangle \simeq 5.2 \times 10^{-3}$ and $\langle\ell\rangle \simeq 0.11$.

The question now is how are reconnection and turbulence properties linked? The magnetic autocorrelation function $C(\boldsymbol{r})=\langle\boldsymbol{b}(\boldsymbol{x}+\boldsymbol{r}) \cdot \boldsymbol{b}(\boldsymbol{x})\rangle /\left\langle b^{2}\right\rangle$ is shown in Fig. 3(b). The correlation length $\lambda_{C}$ is defined as $\int C(r) d r$, a measure of the size of the energy containing islands. The dissipation length, at which the turbulence is critically damped, is defined as $\lambda_{\text {diss }}=R_{\mu}^{-1 / 2}\left\langle j^{2}\right\rangle^{-1 / 4}$, while the Taylor micro-scale, a measure of mean-square gradients, is $\lambda_{T}=\sqrt{\left\langle b^{2}\right\rangle /\left\langle j^{2}\right\rangle}$. In all our simulations we found that the diffusion thickness $\delta$ scales with the dissipation scale, while $\ell$, though broadly scattered, scales with $\lambda_{C}$ (cf. Fig. 3). The main features of this ensemble of reconnecting events, including the key length scales, are evidently controlled by the statistical properties of turbulence.

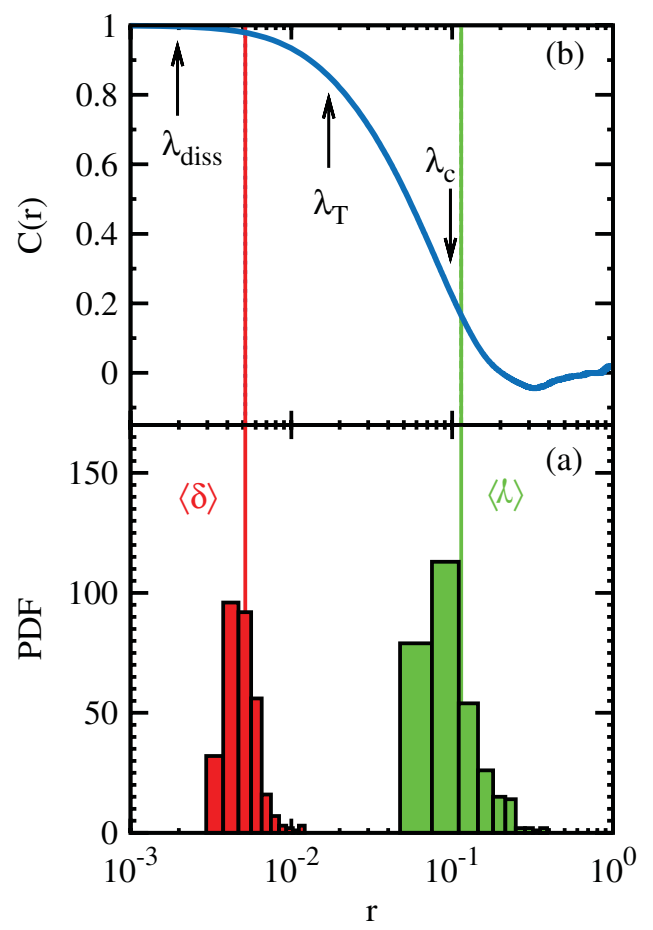

FIG. 3 (color). Bottom (a) histograms of thicknesses ( $\delta$, red bars) and elongations ( $\ell$, green bars). Top (b) magnetic field autocorrelation function (solid blue line), the arrows (left to right) represent, respectively: dissipation scale $\lambda_{\text {diss }}$, Taylor microscale $\lambda_{T}$, and correlation length $\lambda_{C}$. Vertical lines are average values $\langle\delta\rangle$ (red) and $\langle\ell\rangle$ (green).

In order to precisely describe nonsymmetric magnetic field configurations near the $X$ points, the Sweet-Parker analysis of reconnection must be generalized. A recent steady, antiparallel reconnection model [23] allows for asymmetries including unequal upstream magnetic field values $b_{1}$ and $b_{2}$ by analyzing conservation laws in the diffusion region. In the incompressible case the associated reconnection rate is given by

$$
E_{\times}^{\mathrm{th}}=\sqrt{\frac{b_{1}^{3 / 2} b_{2}^{3 / 2}}{R_{\mu} \ell}} .
$$

To understand scaling we evaluated Eq. (7) using several runs (listed in Fig. 4). Figure 4 shows that in all the simulations the reconnection rates are consistent with the prediction given by Eq. (7). In this scenario turbulence plays a crucial role, providing locally the parameters that determine the Sweet-Parker reconnection rate: the lengths and local magnetic field strengths. Apparently, reconnection is an integral part of the overall MHD turbulence cascade process.

To summarize, properties of magnetic reconnection in turbulence have been investigated through high resolution numerical simulations of 2D MHD. Reconnection of different-size magnetic islands occurs simultaneously, spontaneously, and locally. The turbulent cascade produces a distribution of reconnecting islands. Computing the elec- 


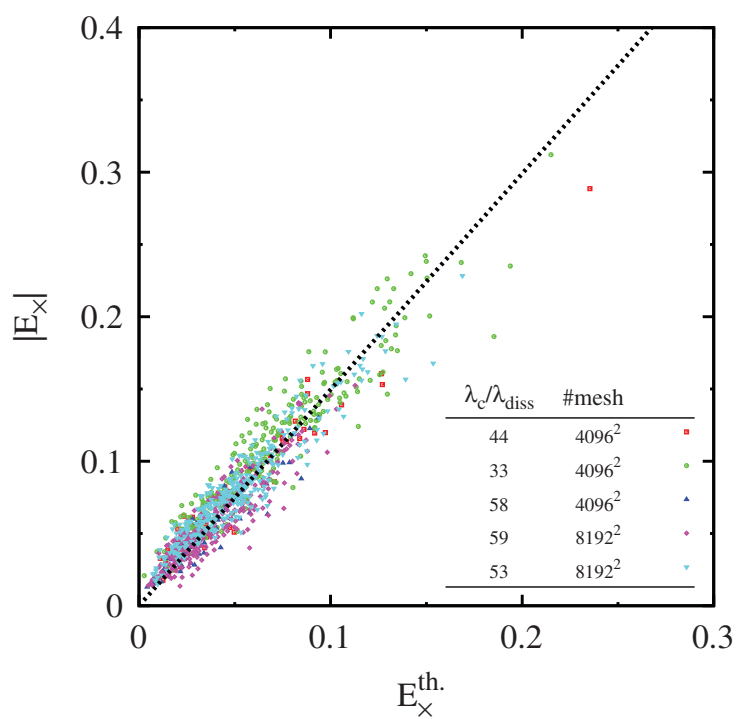

FIG. 4 (color). Computed reconnection rates vs expectation from Eq. (7) [23]. Inset table gives ratio $\lambda_{c} / \lambda_{\text {diss }}$, a measure of the extent of the inertial range, for several runs, along with grid resolution.

tric field at the $X$ points, we see that turbulence produces a broad range of reconnection rates, with values in excess of 0.1 in dimensionless global Alfvén units. In addition, the strongest reconnection rates vary in proportion to $\ell / \delta$, the aspect ratio of the reconnection sites. This scaling appears to differ greatly from classical laminar theories [1], but taking into account the nearby magnetic field produced by the turbulence, a form of generalized Sweet-Parker scaling [23] is restored. These results explain how rapid reconnection occurs in MHD turbulence in association with the most intermittent non-Gaussian current structures, and also how turbulence generates many reconnection sites that have very small rates. Reconnection, like other transport processes, is greatly affected by turbulence [24].

Resistive MHD can give rise to a broad range of reconnection rates in terms of root-mean-square values of the turbulence parameters. Many potential reconnection sites are present, but only a few are selected by the turbulence, at a given time, to display robust reconnection electric fields. In fact, results of the present type may shed light on possible scalings as Reynolds numbers are increased, even though direct computational scalings remain greatly challenging. In particular, we expect that the distribution of reconnection rates can be related to the issue of maintaining finite energy dissipation in the infinite Reynolds numbers.

This new perspective on reconnection may be highly relevant to space and astrophysical applications such as the magnetopause [25], the solar wind [26], and the solar corona [5]. On the basis of the current results, we would expect to find in the turbulent corona and solar wind a broad distribution of size of interacting islands, with a concomitantly broad distribution of reconnection rates. The rates can be determined statistically in terms of mea- surable correlation, Taylor, and dissipation scales. In future studies it may be useful to study additional signatures such as characteristic reconnection flows [26]. Furthermore a useful extension will be to employ models that are suited to low collisionality plasmas, where, for example, anomalous resistivity, Hall MHD, or other kinetic effects, may be important.

Research supported by NSF ATM-0539995, (SHINE) ATM-0752135, NASA NNG06GD47G, NNX07AR48G, and (heliophysics theory) NNX08AI47G. P.D. is a member of CIC-CONICET and thanks grants UBA X429/08, ANPCyT PICT 33370/05, 00856/07.

[1] P. A. Sweet, Electromagnetic Phenomena in Cosmical Physics (Cambridge University Press, New York, 1958); E. N. Parker, J. Geophys. Res. 62, 509 (1957).

[2] B. U. O. Sonnerup et al., in Solar Terrestrial PhysicsPresent and Future, edited by D.M. Butler and K. Papadopoulos, NASA Reference Publication 1120 (NASA, Washington, DC, 1984), Chap. 1.

[3] K. Schindler et al., J. Geophys. Res. 93, 5547 (1988).

[4] V. M. Vasyliunas, Rev. Geophys. Space Phys. 13, 303 (1975).

[5] E. N. Parker, Cosmical Magnetic Fields (Oxford Univ. Press, New York, 1979).

[6] J. B. Taylor, Rev. Mod. Phys. 58, 741 (1986).

[7] T. Sato et al., Phys. Rev. Lett. 41, 1548 (1978).

[8] J. Birn et al., J. Geophys. Res. 106, 3715 (2001).

[9] W.H. Matthaeus and D. Montgomery, Ann. N.Y. Acad. Sci. 357, 203 (1980).

[10] W. H. Matthaeus and S. L. Lamkin, Phys. Fluids 29, 2513 (1986).

[11] H. R. Strauss, Astrophys. J. 326, 412 (1988).

[12] V. Carbone et al., Phys. Fluids A 2, 1487 (1990).

[13] F. Malara et al., Phys. Fluids B 4, 3070 (1992); G. Lapenta, Phys. Rev. Lett. 100, 235001 (2008).

[14] D. Biskamp, Phys. Fluids 29, 1520 (1986).

[15] D. Biskamp, Magnetic Reconnection in Plasmas (Cambridge Univ. Press, Cambridge, 2000).

[16] S. Ghosh et al., Comput. Phys. Commun. 74, 18 (1993).

[17] S. Rana, Surface Topological Data Structures (John Wiley and Sons, Chichester, England, 2004).

[18] R. H. Kraichnan and R. Panda, Phys. Fluids 31, 2395 (1988).

[19] K. Arzner and L. Vlahos, Astrophys. J. 605, L69 (2004).

[20] J.D. Craig and P. G. Watson, Astrophys. J. 516, 924 (1999).

[21] J. C. Dorelli and J. Birn, J. Geophys. Res. 108, 1183 (2003).

[22] J. E. Borovsky and M. Hesse, Phys. Plasmas 14, 102309 (2007).

[23] P. A. Cassak and M. A. Shay, Phys. Plasmas 14, 102114 (2007).

[24] S. A. Orszag, in Fluid Dynamics, edited by R. Ballian and J.-L. Puebe (Gordon \& Breach, N.Y., 1977), p. 235.

[25] A. Retinò et al., Nature Phys. 3, 235 (2007).

[26] J. Gosling and A. Szabo, J. Geophys. Res. 113, A10103 (2008). 